\title{
Frontières
}

\section{Le deuil dans la littérature}

\section{Hélène Reboul}

Volume 16, numéro 2, printemps 2004

\section{Deuil, blessure vive}

URI : https://id.erudit.org/iderudit/1074112ar

DOI : https://doi.org/10.7202/1074112ar

Aller au sommaire du numéro

Éditeur(s)

Université du Québec à Montréal

ISSN

1180-3479 (imprimé)

1916-0976 (numérique)

Découvrir la revue

\section{Citer cet article}

Reboul, H. (2004). Le deuil dans la littérature. Frontières, 16(2), 28-32. https://doi.org/10.7202/1074112ar

\section{Résumé de l'article}

Plusieurs écrivains se sont servis de leur plume pour engager un travail de deuil à la suite du décès d'un proche. Parmi ceux-ci des auteurs très connus, tels que Simone de Beauvoir et Jean-Paul Sartre et d'autres qui le sont moins mais dont l'écrit présente un intérêt non négligeable : Anne Philipe ou Michel Ragon. Dans la mesure où la démarche de ces écrivains, réalisée avec leur sensibilité propre et une certaine liberté d'expression, retrace le vécu avec la personne disparue, ces écrits constituent des autobiographies. Celles-ci sous le terme de Mémoires contribuent également à faire le deuil des décennies passées. Parallèlement, l'auteur de l'article s'engage dans une démarche similaire grâce au choix des ouvrages et à l'intérieur de ceux-ci des circonstances relatées.
Ce document est protégé par la loi sur le droit d'auteur. L'utilisation des services d'Érudit (y compris la reproduction) est assujettie à sa politique d'utilisation que vous pouvez consulter en ligne.

https://apropos.erudit.org/fr/usagers/politique-dutilisation/ 


\section{Résumé}

Plusieurs écrivains se sont servis de leur plume pour engager un travail de deuil à la suite du décès d'un proche. Parmi ceux-ci des auteurs très connus, tels que Simone de Beauvoir et Jean-Paul Sartre et d'autres qui le sont moins mais dont l'écrit présente un intérêt non négligeable: Anne Philipe ou Michel Ragon. Dans la mesure où la démarche de ces écrivains, réalisée avec leur sensibilité propre et une certaine liberté d'expression, retrace le vécu avec la personne disparue, ces écrits constituent des autobiographies. Celles-ci sous le terme de Mémoires contribuent également à faire le deuil des décennies passées. Parallèlement, l'auteur de l'article s'engage dans une démarche similaire grâce au choix des ouvrages et à l'intérieur de ceux-ci des circonstances relatées.

Mots clés : deuil - littérature - mémoiresautobiographie.

\section{Abstract}

A number of authors have used their pen to help their grieving after the loss of someone close. Among these are some well-known authors like Simone de Beauvoir and Jean-Paul Sartre; there are still others who, while less well-known, their writings are not without interest: Anne Philipe or Michel Ragon. Insofar as their efforts to trace, with their unique sensibilities and literary licence, life bereft of a close one, these writings constitute autobiographies. These writings, often known as Memoirs, can help with our mourning of previous decades. The author engages in this process not only through her selection of texts but also through her selection of events told through these texts.

Keywords: grieving - literature memoirs-autobiographies.

\section{LE DEUIL DANS LA LITTÉRATURE}

\section{Hélène Reboul, \\ professeure émérite et fondatrice de l'enseignement en gérontologie à I'Université de Lyon II.}

On dit de tout roman qu'il est plus ou moins autobiographique ; on pourrait élargir cette idée reçue à un certain nombre d'écrits autres. C'est ce que je ferai ici. Peutêtre qu'en avançant dans la vie on serait plus tenté par cette démarche. N'est-ce pas déjà engager une forme de deuil ?

\section{LE DEUIL}

Je suis de la génération qui a vu le deuil dans sa dimension sociale : le port d'habits noirs indiquant la perte d'un proche et pour ce faire la pancarte située à la vitrine du teinturier «Deuil en 24 heures ».

Habitant à proximité d'une entreprise de pompes funèbres, enfant j'admirais la livrée noire dont étaient revêtus les chevaux tirant un corbillard noir décoré de plumeaux situés aux quatre coins. Quotidiennement, je les voyais défiler au sortir de la morgue de l'établissement hospitalier Cochin à Paris.

Dire que pour autant la mort n'était pas redoutée ; ces signes visibles remplaçaientils la parole? Ce qui est certain, c'est qu'il ne serait jamais venu à l'esprit de qui que ce soit à cette époque d'engager, du fait de cette circonstance, une thérapie. Les deuils pathologiques pouvaient faire florès!

La chambre du mort restait le plus souvent en l'état; c'était une manière de maintenir sa présence sans y toucher; cela se pratique encore de nos jours... On n'en parlait pas et c'était ainsi malgré tout ne pas oublier le défunt. On remarquera que quel que soit le sexe de la personne morte la dimension neutre devient normale!

Écrire à propos de la mort d'une personne proche favorise le travail psychique du deuil qui aide à s'éloigner d'elle tout en mesurant ce qui a été vécu avec elle. Les écrivains ont eu recours à ce procédé ; ainsi la littérature nous fournit-elle un certain nombre d'exemples qui ne sont pas dénués d'intérêt. Cependant, il paraît nécessaire de distinguer des ouvrages écrits par des femmes et d'autres écrits par des hommes, chaque sexe manifestant sa sensibilité différemment sans parler de la personnalité propre. L'écriture donne aussi une certaine liberté.

\section{LA LITTÉRATURE}

Cette dimension m'intéresse tout particulièrement parce que j'en ai fait un usage abondant dans le cadre de mon enseignement universitaire. Cela est survenu à partir du moment où l'université française a accueilli en son sein des professionnels pour se former alors qu'ils ne détenaient pas de diplômes supérieurs, ni parfois le baccalauréat; cela survenait après mai 1968 et de manière plus concrète avec la Loi de 1971 sur la formation continue et l'éducation permanente. Face à leur désir d'apprendre, il ne convenait pas, à mon sens, de les décourager notamment avec des ouvrages théoriques auxquels ils n'avaient pas recours habituellement et aussi fallaitil faire le pari que par ce biais ils s'adonneraient plus facilement à la lecture.

Dans le cadre de mon enseignement de gérontologie psychologique et sociale, du D.U.G.S. (Diplôme d'université de gérontologie sociale créé en 1975), j'ai proposé que chaque étudiant au cours de la première année lise cinq romans traitant du vieillissement, de la vieillesse et de la mort. En outre, je demandais de faire des investigations sur l'auteur et sur les circonstances qui l'avaient conduit à écrire cet ouvrage. 
Puis, il convenait d'établir des parallèles entre les situations décrites dans le livre et des exemples personnels et / ou professionnels. Enfin, la validation se réalisait en groupe avec ceux qui avaient lu le même écrit. Cela permettait alors à chacun de découvrir qu'à partir d'un même livre on pouvait faire une lecture différente et ceci en fonction de sa propre personnalité et de son histoire familiale. Cette manière de pratiquer pouvait aussi donner aux autres étudiants le désir de lire les livres ainsi présentés.

Ce constat devait ultérieurement faire comprendre, par rapport à des paroles dites, qu'on n'en retienne pas les mêmes données que l'on pourrait interpréter différemment. Deux auteurs ont principalement éveillé mon intérêt, Simone de Beauvoir et JeanPaul Sartre, mais d'autres prendront rang également.

On verra selon les ouvrages que le deuil réalisé par l'écrit ne concerne pas obligatoirement des pertes semblables.

\section{QUELQUES EXEMPLES}

Simone de Beauvoir que certains ont appelé «la grande sartreuse... » parce que compagne de Jean-Paul Sartre, écrivain féministe, existentialiste et humaniste selon les qualificatifs couramment indiqués la concernant, a écrit un ouvrage dont le titre est en prise directe avec la mort : Une mort très douce, celle de sa mère qui fut « douce » grâce à l'accompagnement constant par ses deux filles tout au long de son hospitalisation. Ouvrage publié en 1964. L'auteure avait 56 ans à la mort de sa mère. Elle relate la maladie, l'agonie et la mort de celle-ci. Il faut savoir que tous les écrits de cette auteure l'ont été à partir du journal qu'elle tenait quotidiennement. Ce qui explique les si nombreux détails dont elle a illustré cet ouvrage ; il faut convenir que le fait de rester assise auprès d'une malade rend attentif aux moindres faits et réactions. En même temps, il y a un retour sur le passé vécu par ces deux femmes dont les relations n'étaient pas des meilleures! La mère reprochant à sa fille d'être athée, de ne pas avoir engagé sa vie dans les liens du mariage et, par voie de conséquence, de ne pas lui avoir donné de petits-enfants qui l'auraient prolongée...

Après avoir repris le déroulement de l'existence de sa mère de manière à la situer pour le lecteur et entrepris le récit de la maladie qui la conduirait à la mort, Simone de Beauvoir découvre:

Pour moi, ma mère avait toujours existé et je n'avais jamais sérieusement pensé que je la verrais disparaître un jour, bientôt. Sa fin se situait, comme sa naissance, dans un temps mythique. Quand je me disais : elle a l'âge de mourir, c'étaient des mots vides, comme tant de mots.

Pour la première fois, j'apercevais en elle un cadavre en sursis (p. 26).

Impensable jusqu'alors pour elle d'envisager que celle qui donne la vie soit destinée à la mort !

Et puis, les détails ne manqueront pas sur le corps décharné de sa mère; d'une fracture osseuse pour laquelle elle était hospitalisée, on découvre après coup un cancer qui n'avait pas été diagnostiqué en temps voulu! Cette atteinte gravissime contribue à transformer l'image qu'elle avait de sa mère auparavant.

Autre passage intéressant mentionnant la difficulté de parler de sa mort avec une personne en période terminale et qui ne s'en rend pas compte ; le malaise cache souvent le mensonge. En effet, un jeune jésuite vient lui rendre visite:

Charles Cordonnier n'est resté que cinq minutes. Il a parlé des déjeuners auxquels chaque semaine son père invitait maman: "Je compte bien vous revoir boulevard Raspail un de ces jeudis. » Elle l'a regardé, incrédule et navrée: «Tu penses que j'y retournerai ? » Jamais encore je n'avais vu sur son visage un tel air de malheur: ce jour-là, elle a deviné qu'elle était perdue (p. 95).

C'est à partir de ce moment qu'elle fera des cauchemars avec la notion de chute dans un trou et de draps bleus... Elle aurait convenu à ce moment-là d'aider sa mère à engager le deuil de son existence pour s'acheminer vers la fin de sa vie. À cette époque, on préférait cacher la réalité et on s'engageait dans une démarche basée sur le mensonge, qui était le plus souvent pernicieuse pour les divers interlocuteurs.

Cette maladie qui a duré un certain temps a permis aux deux filles de madame de Beauvoir de se préparer, elles, à la mort de leur mère, et cependant à la fin de l'ouvrage Simone écrit:

$\mathrm{Si}$ je rencontrais une femme de cinquante ans accablée parce qu'elle venait de perdre sa mère, je la tenais pour une névrosée: nous sommes tous mortels ${ }^{1}$; à quatre-vingts ans on est bien assez vieux pour faire un mort...

Mais non. On ne meurt pas d'être né, ni d'avoir vécu, ni de vieillesse. On meurt de quelque chose (p. 156-157).

Et de conclure : «Il n'y a pas de mort naturelle : rien de ce qui arrive à l'homme n'est jamais naturel puisque sa présence met le monde en question. »
De tels propos tenus il $\mathrm{y}$ a près de quarante ans demeurent aujourd'hui d'une actualité cuisante après, entre autres, les effets de la canicule en France au cours de l'été 2003 !

Par ailleurs, la prise de conscience faite par Simone de Beauvoir à l'occasion du décès de sa mère la touche d'autant plus que le «paratonnerre par rapport à la mort » que constituait cette dernière disparaissait et la mettait à découvert. Mais, à l'époque, Jean-Paul Sartre était vivant et, durant l'accompagnement de sa mère, elle trouvait un soutien auprès de lui.

À son tour, la mort a emporté le grand homme. Elle écrit alors La cérémonie des adieux qui date de 1981. À l'époque, Simone de Beauvoir a 73 ans. L'ouvrage est dédicacé : «À ceux qui ont aimé Sartre, l'aiment, l'aimeront» (p. 9).

Avec ce titre, apparemment bien approprié pour la mort de Sartre, elle citait de fait une phrase qu'il avait prononcée lorsque Sylvie, la fille adoptée de Simone, était venue la chercher pour partir toutes les deux en vacances! Utilisant cette phrase, elle le rend encore présent! Il faut savoir aussi que son compagnon est mort en milieu hospitalier et qu'à ce moment-là elle s'est glissée dans le lit ; étant donné la contamination possible par le staphylocoque doré, le personnel lui a demandé de se mettre sur les couvertures. La séparation était difficile. Mais restant de manière si proche de lui, elle devait prendre conscience du froid qui s'installait dans ce corps.

La Préface explicite ce que représente ce livre par rapport à Sartre: «Voici le premier de mes livres - le seul sans doute que vous n'aurez pas lu avant qu'il ne soit imprimé. Il vous est tout entier consacré et ne vous concerne pas» (p. 13). Et d'expliquer qu'elle destine cet ouvrage aux amis de Sartre pour qu'ils puissent mieux connaître ses dernières années. Ce faisant, elle engage le deuil d'une cinquantaine d'années partagées avec lui.

Malheureusement, ceux qui ont admiré l'homme, le philosophe, lui ont reproché d'avoir dévoilé les misères physiques qu'il avait endurées avant de mourir: elles entachaient l'image qu'ils auraient voulu garder de lui ! Et alors, pour ces derniers quel deuil possible?

Pour nous qui lisons cet ouvrage apparaît la qualité de présence et d'attention que Simone de Beauvoir lui a manifestée et rend compte de l'importance du lien qui les unissait depuis si longtemps.

L'attachement à sa mère diffère bien de celui qu'elle manifesta pour Sartre; mais, dans les deux cas, l'ouvrage rédigé a facilité le processus d'acceptation de la disparition de chacun d'eux en reprenant les 
événements vécus ensemble, tout au long de la vie avec Sartre et au cours de la maladie avec sa mère.

Une autre forme d'écrit que Simone de Beauvoir a utilisé concerne son passé et sa vie relationnelle très riche, à savoir les Mémoires, qui constituent la reprise du déroulement de son existence. Pour nous, ils constituent une source incommensurable d'informations sur elle, sur Sartre, également sur leurs amis et sur l'histoire collective ainsi traversée tout au long de ces années.

Il est à noter que le mot "Mémoires", terme utilisé qui concerne cette forme littéraire, prend une majuscule. Est-ce une manière de magnifier la personne qui a recours à cette écriture, ainsi que la vie qu'elle a menée et dont elle rend compte parfois avec beaucoup de courage ? Est-ce la reconnaissance de ce parcours ? Jusqu'à présent, je n'ai pas trouvé d'explication plausible ni de personne pouvant m'instruire à ce sujet !

Ces ouvrages sont au nombre de quatre : trois sont bien connus et le dernier beaucoup moins. Mémoires d'une jeune-fille rangée (1958), La Force de l'âge (1960), La Force des choses (1963), Tout compte fait (1972). Du premier écrit, on peut noter qu'il paraît quand Simone de Beauvoir a 50 ans (elle est née en 1908). Ce chiffre synthétise le milieu de la vie dont la prise de conscience incite à établir le bilan de ce qui a été vécu jusqu'alors; il présente un certain aspect attractif pour les esprits cartésiens. Cette démarche est plus familière aux femmes qu'aux hommes comme les recherches menées avec mes étudiants nous l'avaient fait découvrir.

Cet ouvrage inaugure une série puisque le suivant survient deux ans après et le suivant trois ans encore après. Alors que, pour le quatrième, il y aura quasiment une décennie qui suivra. Mais, entre-temps, est paru le gros volume sur La Vieillesse (1970) pour lequel on lui aurait reproché de ne pas avoir parlé de sa propre vieillesse. Elle reconnaît que dix ans ont passé avec d'autres événements. Cependant, elle s'approche des 65 ans qui, à l'époque, marquent déjà une avancée certaine dans le vieillissement et modifie son attitude par rapport aux autobiographies précédentes pour lesquelles elle a respecté l'ordre chronologique.

Après avoir donné ces explications, elle termine ainsi le Prologue de Tout compte fait :

Je n'ai plus l'impression de me diriger vers un but mais seulement de glisser inéluctablement vers ma tombe. Alors il ne m'est plus nécessaire de prendre pour fil conducteur le déroulement du temps; dans une certaine mesure je tiendrai compte de la chronologie; mais c'est autour de certains thèmes que j'organiserai mes souvenirs (p. 10).

Glissant vers la fin de sa vie signifie que ses écrits précédents l'ont aidée à réaliser un certain deuil de son existence passée. Dans l'ouvrage écrit par Chantal Moubachir sur Simone de Beauvoir, publié en 1972 également, elle rend compte d'une parole de celle-ci :

Moi, l'évidence de mon vieillissement m'a frappée entre 1958 et 1962 . [C'est le temps où elle inaugure la série des Mémoires.] Écœurée par les crimes qui se commettaient au nom de la France, je me suis retournée avec nostalgie vers mon passé et j'ai réalisé que, sur beaucoup de plans, il me fallait lui dire un définitif adieu (p. 186).

Ainsi confirme-t-elle que le processus de deuil est réellement en cours de réalisation.

Il est intéressant de noter que Francis Jeanson, un des biographes de Sartre, ait dans son ouvrage Simone de Beauvoir ou l'entreprise de vivre (1966) utilisé l'expression " tous comptes faits» quand il évoque le premier livre des Mémoires «cette œuvre est d'abord pour moi une aventure personnelle» (p. 11).

Ces thèmes mentionnés concernent, entre autres, les événements politiques, puisque l'on sait que Sartre et elle étaient très engagés. Bien évidemment, elle parle de ceux qui ont été proches ; elle rend compte de déplacements, etc. Mais l'ensemble est abordé avec plus de distance et de profondeur que dans les volumes précédents qui ressemblaient davantage à une chronique. Et de fait, ce dernier ouvrage qu'elle n'était pas loin de considérer comme tel (il y eut seulement après La Cérémonie des Adieux) pouvait induire une remise en question du lecteur. Est-ce que cela aurait contribué à moins susciter l'intérêt du lecteur?

Ainsi donc cette succession d'ouvrages de Simone de Beauvoir cités ici montre que l'écrivain peut, par des écrits dont la forme diffère quelque peu, les utiliser à une fin semblable, à savoir celle d'engager un travail de deuil. Il est évident que pour toute personne la mise par écrit de ses mémoires confère aux lecteurs une image de soi que l'on souhaite laisser aux siens comme à ses contemporains. Cependant, cette démarche provoque obligatoirement une relecture de son existence, démarche dont on devient le premier bénéficiaire. Les divers ouvrages cités ici destinés à évoquer une personne défunte jouent pour l'écrivain un rôle similaire à celui des Mémoires

\section{AUTRES EXEMPLES}

Comme je l'indiquais au début de cet écrit, la référence suivante émane d'un écrivain dont les écrits ont concerné divers domaines qui l'ont fait connaître à savoir les arts, l'histoire notamment, cette dernière sous forme de romans ; mais il a également écrit un ouvrage sur sa mère après la mort de celle-ci. Il s'agit de Michel Ragon, originaire de l'Ouest de la France, de la Vendée plus précisément, mais résidant à Paris. Cet ouvrage s'intitule L'accent de ma mère. Il fut publié en 1980, l'année qui a suivi le décès de celle qui lui a donné le jour; il a 56 ans. Il s'agit donc de l'accent vendéen puisque lui et sa mère sont nés à Fontenayle-Comte. Veuve d'un militaire alors que lui a 8 ans, elle partira avec son fils à Nantes, cette ville qui pour elle sera son nouveau point d'ancrage.

Il « montera » à Paris à 21 ans. Lui et sa mère ont en commun l'amour de la lecture ; pour elle, c'est un moyen de s'échapper de la réalité difficile au gré des emplois et, par la suite, des maisons de retraite où elle demande asile en hiver, la lecture alimentant ses rêves. Pour lui, la lecture constitue une ouverture, un moyen de se cultiver: il est connu comme étant autodidacte.

Tant que Michel Ragon a vécu à Nantes il ne percevait pas l'accent vendéen. C'est après un grand nombre d'années passées à Paris au cours desquelles il a vraisemblablement perdu cet accent qu'un jour étant au téléphone avec sa mère, il perçoit sa manière de parler liée à son terroir. Il mesure alors la distance existant entre eux deux : il ne se rappelait plus cette sonorité de sa voix, qui lui paraît alors quelque peu étrange : "Cet accent traînant de l'Ouest, cet accent paysan qui lui fait la voix grasse, comme imprégnée de terre humide. » C'est Henri Quéffelec qui relève cette phrase si parlante pour lui dans l'article qu'il dédie à cet ouvrage, article situé dans un magnifique livre consacré à Michel Ragon. Et de remarquer que cet écrit agit comme un révélateur d'un aspect ignoré de son histoire et de sa personnalité.

Ce décalage fait prendre alors conscience à l'auteur de la différence de culture survenue entre lui et sa mère: la culture paysanne qui fut la sienne à l'origine et qui est restée celle de sa mère et la culture citadine de Paris adoptée par lui. Alors apparaît le chemin qu'il a parcouru.

Il accompagnera sa mère alors en maison de retraite jusqu'à sa mort; il lui cachera la gravité de son état: elle est atteinte d'un cancer. Il la protège ainsi comme si elle était devenue une petite fille et lui l'adulte. De nouveau, c'est la mort de l'être cher qui provoque la mise à l'écrit. Ici le titre de l'ouvrage indique une spécificité que la disparition de la mère fait émerger, l'aidant, lui, à prendre conscience de la distance qui s'était instaurée entre elle et lui, chacun vivant dans deux univers si différents, voire presque opposés. De fait, elle 


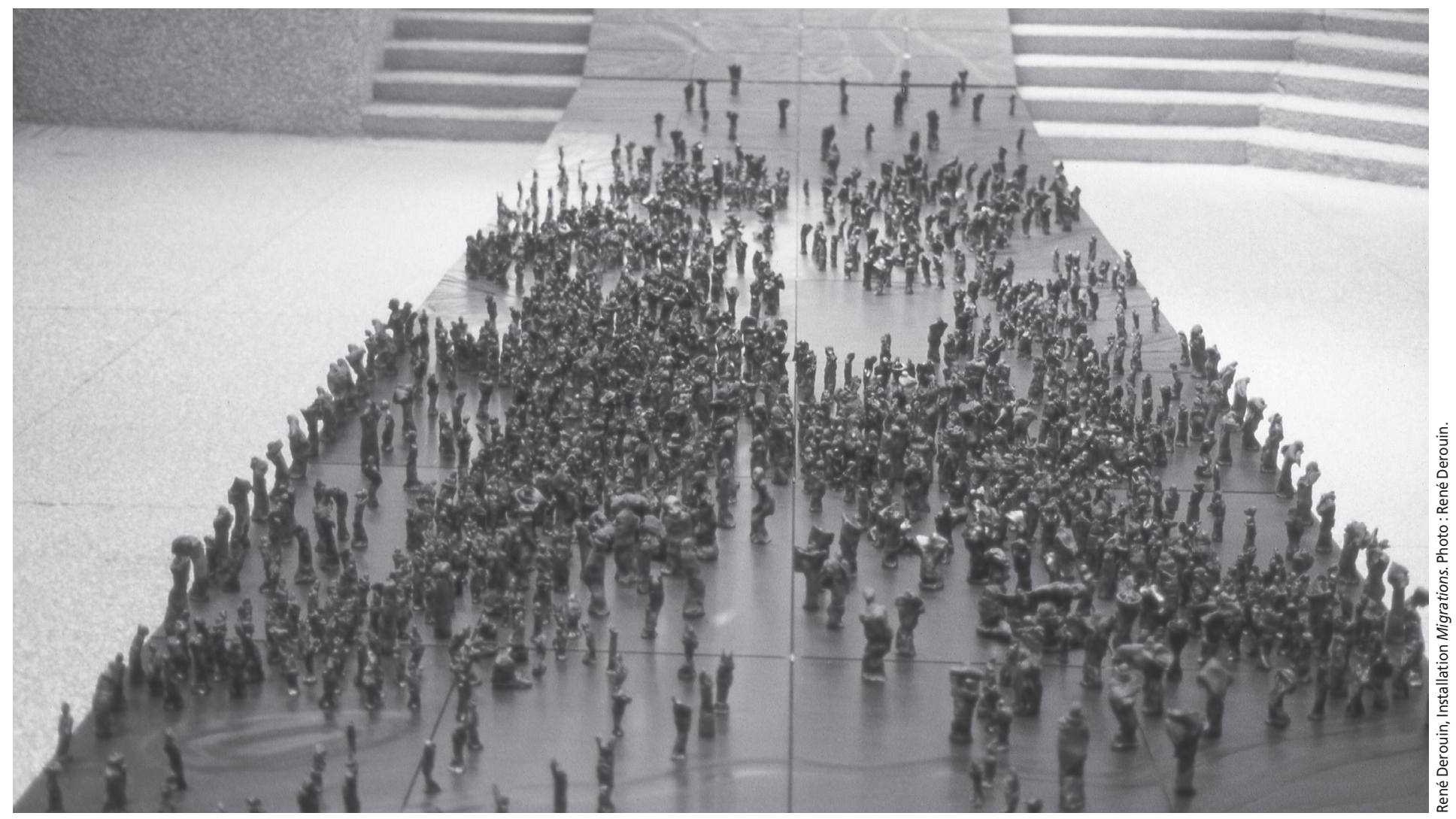

lui écrivait toutes les semaines; ce qui lui permettait une forme de mutisme sonore...

On peut rappeler que Françoise Xénakis, écrivain et critique littéraire, considère cet ouvrage comme un bouleversant livre d'amour. L'avis de cette femme constitue un compliment de grande valeur.

Un autre ouvrage, cette fois-ci écrit par une femme, concerne le deuil du mari. Le temps d'un soupir (1963) d'Anne Philipe, la veuve du célèbre acteur Gérard Philipe. Cet ouvrage très émouvant constitue « une méditation sur l'amour et sur la mort » tel qu'il est présenté sur la jaquette du livre. Anne Philipe était ethnologue, aussi son métier l'a-t-il rendue sensible à des détails de la vie courante aussi bien qu'aux paysages. Cet écrit est provoqué par la mort de son mari à la suite d'un cancer qui a donné lieu à une intervention chirurgicale sans espoir. Il est mort à leur domicile. «[...] de souvenir en souvenir, je glisse, en tournant la tête vers l'oreiller que je continue à poser chaque soir à ma droite, à la vision de ton visage mort... au moment où la vie te quitta»(p. 9). Dès la première page, l'auteure évoque la fin de vie de son époux.

Elle décrit les heures qui ont suivi au cours desquelles elle demeura auprès de lui, comme l'avait fait Simone de Beauvoir auprès de Sartre jusqu'au moment où elle constata: "La mort nous séparait pour l'éternité» (p. 10). Et de se poser un peu plus loin la question «Faut-il accepter un futur dont tu es absent? » tout en avouant «Jusque là je n'avais jamais été intéressée par la mort. Je ne comptais pas avec elle. Seule la vie importait. La mort? Un rendez-vous inéluctable et éternellement manqué puisque sa présence signifie notre absence » (p. 12).

C'est donc à partir de là qu'elle entreprend ce chemin réalisé et poursuivi. Les pages suivantes sont émaillées de souvenirs de la vie partagée avec lui. "Le bonheur nous pénétrait comme une odeur, nous l'oubliions parfois tant nous étions privilégiés » (p. 94). Mais ce couple avait deux enfants auxquels il faut annoncer l'irrémédiable.

Quand je lisais ce livre, à l'époque j'avais déjà mes cinq enfants, et le passage où elle relate l'échange avec ses propres enfants me marqua profondément. L'identification à Anne Philipe se faisait naturellement. J'admirais la manière dont elle s'y était prise. "J'essayais de te lier à la vie. Il s'est transformé, disais-je, il est devenu deux arbres et des fleurs; les abeilles le butinent; elles font du miel et nous mangeons du miel et comme cela tout recommence. » Ainsi, elle dépassait l'immobilisme de la mort fournissant à ses enfants un support dynamique. Elle relate alors la réaction de chacun d'eux «Beau comme il était, m'a dit l'un d'un air épanoui, il a du faire de belles fleurs! L'autre a réfléchi, silencieux. Le lendemain, il est venu vers moi. En somme, quand nous mangeons du miel, nous mangeons un peu de l'homme, m'a-t-il dit» (p. 98).
J'ai toujours gardé en mémoire ce passage. Je le citais dans mes interventions, partant du fait que, par la manière dont on parle de la mort avec les enfants en répondant à leur demande, on arrive à atténuer l'angoisse que peut générer cet événement et les aider à engager le deuil de cette personne qui est partie.

Après une multitude de détails, plus touchants les uns que les autres qui font de ce livre, petit par son volume mais grand par sa qualité d'humanité, elle le termine en se laissant aller à rêver : elle souhaite sa présence; mais la réalité est là. Elle clôt l'ouvrage ainsi « Je veux me sauver, non me délivrer de toi » (p. 143). Telle est la dernière phrase.

Cette pensée magistrale montre qu'à travers l'écrit elle a entamé le travail du deuil et qu'elle a saisi l'importance de la démarche.

À la mort de sa mère, Anne Philipe écrivit un autre ouvrage: Je l'entends respirer (1984). Elle montre une fin de vie sereine telle qu'elle se déroule pour bien des personnes vieillies vivant à leur domicile et dont la vieillesse se passe remplie par la lecture, par la musique... et la pensée pour les autres, « une solitude peuplée » comme la décrivait Jean Guiton.

De plus, le quotidien est vécu avec un animal, en l'occurrence un chat. Évidemment, cette vieille femme bénéficiait d'un accompagnement professionnel parant aux gestes difficiles et lui permettant de 
rester dans son cadre de vie. Sa fille ne la négligeait pas non plus.

Poursuivant et pour clore ces références, ajoutons Les Mots de Jean-Paul Sartre que l'on peut percevoir comme un livre autobiographique: la reprise de vie se base à partir de l'absence du père mort alors que l'auteur avait quelque dix-huit mois.

Philippe Lejeune dans Obliques consacré à Sartre indique dans son analyse sur Les Mots que le philosophe avait commencé l'écriture de ce livre dans les années 1953-1954 ; rappelons que le premier livre de Mémoires de Simone de Beauvoir parut en 1958 ! Sartre attendait aussi que sa mère n'ait pas à lire dans cet ouvrage ce qui les concernait, elle et son second mari avec qui son fils ne s'entendait pas des mieux... Mais, finalement, il parut avant son décès et elle l'accepta.

Ceci montre que ces livres autobiographiques, dans la mesure où ils ont trait aussi à la vie des personnes de l'entourage de l'auteur, induisent parfois des restrictions ou des autocensures!

Le début de cet écrit évoque sa mère Anne-Marie, de la lignée du docteur Albert Schweitzer. À 20 ans, elle épouse en 1904 un officier de marine «déjà rongé par les fièvres de Cochinchine ». Ils ont rapidement un enfant, Jean-Paul. Elle soigne avec dévouement l'un et l'autre. Mais la fatigue tarit le lait. L'enfant à neuf mois est alors confié à une nourrice, c'est-à-dire éloigné de ses parents, ce qui nuit à sa santé. «À vingt ans, sans expérience ni conseils, ma mère se déchirait entre deux moribonds » (p. 9).

À la mort du mari, la veuve récupère son enfant, qui alors guérit. Sans ressource, elle se rend chez ses parents, Karl et Louise Schweitzer qui vivent à Meudon à proximité de la capitale et, en 1911, ils déménagent à Paris au Quartier Latin, lui, ayant été enseignant. Les deux, mère et fils, demeureront chez les grands-parents encore quelques années. C'est le grand-père retraité qui reprenant du service assure le quotidien et devient un substitut paternel pour son petit-fils, lui fournissant le soubassement intellectuel qui alimentera son devenir, lui donnant aussi la possibilité de s'identifier à un homme.

Sa mère très grande lui apparaissait comme un géant, à lui qui est toujours resté petit. Il insistera beaucoup sur ce qu'a produit sur lui la mort de ce père à 30 ans. Et de constater: "J'ai laissé derrière moi un jeune mort qui n'eut pas le temps d'être mon père et qui pourrait être, aujourd'hui mon fils» (p. 11).

Ce qui peut expliquer que le livre Les Mots comprennent deux parties à l'appellation bien symptomatique: «Lire» pour la première, «Écrire » pour la seconde et de constater «J'ai commencé ma vie comme je la finirai sans doute: au milieu des livres» (p. 29).

À la fin de l'ouvrage, il évoque à nouveau sa mort : "J'ai perdu mes chances de mourir inconnu. » Quand il écrit cet ouvrage, il est quinquagénaire. Il est vrai que, dans les années 1960, l'espérance de vie était moins élevée que maintenant. Cependant, Les Mots, censés évoquer son cheminement qui le conduit à écrire, sont marqués par l'absence du père: "Je me suis mis tout entier à l'œuvre pour me sauver tout entier » (p. 212-213). Les psychanalystes lui ont dit que l'absence du père l'a privé du sur-moi, c'est-à-dire du cadre plus ou moins autoritaire influant sur le comportement à partir de valeurs morales.

$\mathrm{Au}$ terme de ces rencontres marquées par la mort dans le cadre de l'étude du deuil, un certain nombre de remarques s'imposent:

- tous ces auteurs sont des Français,

parvenus à près de la moitié de leur existence;

- je les ai choisis ayant écrit les œuvres retenues aux environs de la seconde

moitié du XX $\mathrm{XX}^{\mathrm{e}}$ siècle, parce que ce sont des auteurs qui m'ont alors marquée et que j'ai bien étudiés.

Pour ce travail de présentation des œuvres et leur analyse, je me suis appuyée sur l'ouvrage de Jean Bellemin-Noel, Psychanalyse et Littérature. Pour lui, il est important « de comprendre que les œuvres font partie de la littérature [...] et de trouver les raisons pour lesquelles ces œuvres dépassent leur auteur, leur époque, leur cercle linguistique» (p. 7).

Le thème du deuil est en quelque sorte fédérateur par rapport aux différentes œuvres traitées ici. Les deux femmes, Simone de Beauvoir et Anne Philippe, abordent la mort de leur compagnon et de leur mère, dans un ordre différent du fait des événements.

Lidentification à la mère se fait en fonction de la qualité des relations et des circonstances de la fin de vie. Pour la première, Une mort très douce m'apparaît aussi comme une tentative de réconciliation post mortem. De fait, cet ouvrage est dédié à sa sœur. Cela peut d'autant mieux se comprendre que la sœur de Simone, Poupette, avait eu une bonne relation avec leur mère. Donc cet accompagnement commun a fait ressortir la différence. Encore que Madame de Beauvoir appréciait d'avoir auprès d'elle ses deux filles ensemble; ce qu'elle n'avait jamais eu auparavant.

En ce qui concerne l'écriture des Mémoires ou des ouvrages autobiographiques, le récit de vie intègre d'autres personnes. Cependant, pour Michel Ragon, comme pour Jean-Paul Sartre, la référence aux géniteurs, notamment à la mère, prend un caractère intimiste. Par contre, chez Michel Ragon, le père occupe une place réduite et, pour Sartre, il m'apparaît en permanence en filigrane. Cette absence-présence du père n'a-t-elle pas poussé ce philosophe à jouer le rôle de "père spirituel» ou de leader auprès de ses compagnons plus jeunes?

Quoi qu'il en soit, le travail du deuil engagé aide à envisager l'avenir ou le devenir. Il lui confère des bases solides, des étayages psychiques.

Enfin, j'éclairerai d'une part l'articulation que Sartre a réalisé dans l'ouvrage présenté ici entre le Lire et l'Écrire et d'autre part la manière dont j'ai rendu compte des ouvrages que j'ai cités par cette intéressante citation de Jean Bellemin-Noel : "Qu'estce que je lis quand je lis? Qu'est-ce que lit un écrivain quand il écrit? La réponse est la même: dans l'œuvre littéraire quelle qu'elle soit, qu'on la produise ou qu'on la consomme, on se lit d'abord soi-même » (p. 36).

N'est-ce pas ce que j'ai fait ici? N'ai-je pas fait le deuil du milieu de ma vie quand je relisais ces ouvrages les citant ici? Oui et encore beaucoup plus!

\section{Bibliographie}

BEAUVOIR, Simone de (1970). Une mort très douce, Paris, Presses universitaires de France, coll. «Que sais-je ? », n 2747, 159 p.

BEAUVOIR, Simone de (1972). Tout compte fait, Paris, Gallimard, $516 \mathrm{p}$.

BEAUVOIR, Simone de (1981). La Cérémonie des adieux, Paris, Gallimard, NRF, 159 p.

BELLEMIN-NOEL, Jean (1978). Psychanalyse et littérature, Paris, Presses universitaires de France., coll. "Que sais-je ? », n 1752, $128 \mathrm{p}$.

JEANSON, Francis (1966). Simone de Beauvoir ou l'entreprise de vivre, Paris, Seuil, $302 \mathrm{p}$.

LEJEUNE, Philipe (s.d.). «L'autobiographie parlée », Obliques, nº spécial 18-19, p. 97-116.

MOUBACHIR, Chantal (1972). Simone de Beauvoir ou le souci de différence, Paris, Seghers, coll. «Philosophes de tous les temps », $191 \mathrm{p}$.

PHILIPE, Anne (1963). Le temps d'un soupir, Edit. Julliard, $115^{\mathrm{e}}$ mille, $144 \mathrm{p}$.

QUÉFFELEC, Henri (1980). L'accent de ma mère, dans Autour de Michel Ragon (1984), Musée des Beaux-Arts de Nantes, p. 128-134.

SARTRE, Jean-Paul (1964). Les Mots, Paris, Gallimard, $216 \mathrm{p}$.

\section{Note}

1. Rappelons qu'en 1946 elle avait publié Tous les hommes sont mortels. 